\title{
Role of non-specific interactions in the
}

\section{phase-separation and maturation of}

\section{macromolecules}

\author{
Rakesh Krishnan, Srivastav Ranganathan ${ }^{1}$, Samir K Maji, and Ranjith \\ Padinhateeri* $^{*}$ \\ Department of Biosciences and Bioengineering, Indian Institute of Technology Bombay, \\ Mumbai 400076 \\ E-mail: ranjithp@iitb.ac.in
}

\begin{abstract}
Phase separation of biomolecules could be mediated by both specific and nonspecific interactions. How the interplay between non-specific and specific interactions along with polymer entropy influences phase separation is an open question. We address this question by simulating self-associating molecules as polymer chains with a short core stretch that forms the specifically interacting functional interface, and longer non-core regions that participate in non-specific/promiscuous interactions. Our results show that the interplay of specific (strength, $\epsilon_{\mathrm{sp}}$ ) and non-specific interactions (strength, $\epsilon_{\mathrm{ns}}$ ) could result in phase separation of polymers and its transition to solidlike aggregates (mature state). In the absence of $\epsilon_{\mathrm{ns}}$, the polymer chains do not dwell long enough in the vicinity of each other to undergo phase separation and transition
\end{abstract}

\footnotetext{
${ }^{1}$ SR was affiliated with IIT Bombay while this work was conceptualized, performed and analyzed. His current correspondence address is: 12 Oxford Street, Department of Chemistry and Chemical Biology, Harvard University, MA, USA. email: srivastav_ranganathan@fas.harvard.edu
} 
into a mature state. On the other hand, in the limit of strong $\epsilon_{\mathrm{ns}}$, the assemblies cannot transition into the mature state because they get kinetically trapped, suggesting an optimal range of interactions favoring mature multimers. In the scenario where only a fraction $\left(\mathrm{N}_{\text {frac }}\right)$ of the non-core regions participate in attractive interactions, we find that a slight modifications to either $\epsilon_{\mathrm{ns}}$ or $\mathrm{N}_{\text {frac }}$ can result in a dramatically altered self-assembled states. Using a combination of heterogeneous and homogeneous mix of polymers, we establish how this interplay between interaction energies dictates the propensity of biomolecules to find the correct binding partner at dilute concentrations in crowded environments.

\section{Introduction}

Liquid-liquid phase separation plays a key role in regulating Spatio-temporal organization of biomolecules in the cell. Proteins and/or RNA are known to phase-separate into polymerdense phases known as membraneless organelles (MLOs), often correlated to external stresses or a certain phase of cell division. ${ }^{1,2}$ Interestingly, the local concentration of proteins within these compartments is 50-100 fold higher than their bulk concentrations. ${ }^{3}$ Given that the bulk concentration of phase-separating components could be in the low nanomolar range, this gives rise to an interesting hypothesis - could liquid-like phase-separated compartments facilitate biochemical reactions within this 'polymer-rich' phase by increasing the likelihood of key functional interactions? This gains importance in the context of two interaction partners $(\approx$ $\mathrm{nM}$ concentrations) meeting in space and locking into place to form functional complexes; this is a non-trivial problem. Not only does the low bulk concentration of interacting components make their diffusing-limited meeting unlikely, these dynamic molecules must then undergo further structural reorganization to enable key 'native' interactions for their functionality.

Phase-separated MLOs such as P-bodies and stress granules are known to be stabilized by a network of interactions between specifically interacting domains which bind to unique interaction partners. Interestingly, in addition to these specifically interacting domains, 
protein sequences also bear regions with no known functional interactions. Protein-protein interaction studies suggest that large portions of the interactome are made up of promiscuous or "noisy" interactions with no known function. ${ }^{4-8}$ Therefore, could non-specific interactions play a role in assembly of liquid-like polymer-dense phases en route to formation of specific contacts? Is there a regime of interaction strengths that results in non-specifically driven phase-separation leading to formation of specific contacts?

Coarse-grained computational models have been extensively employed to study various aspects of biomolecular self-assembly and phase-separation. Typical approaches in coarsegrained simulations include the modeling of proteins as lattice polymers, ${ }^{9-11}$ patchy particles $^{12,13}$ and spheres/beads. ${ }^{14}$ Deeds et al. simulated lattice polymers and suggested the existence of a narrow range of temperatures, where the formation of specific complexes is favored despite the presence of promiscuous interactions. ${ }^{9}$ Saric et al. employed a patchy sphero-cylindrical representation of proteins to establish the key role of non-specific interactions in promoting the nucleation of amyloid fibrils. ${ }^{13}$ Osmanovic and Rabin employed phenomenological Monte Carlo simulations to demonstrate how non-specific interactions could assist the formation of specific complexes using a group of interacting particles. ${ }^{14}$ Another class of computational models is the sticker-spacer model, where the stickers are regions with high attractive interactions and spacer residues, on the other hand, are beads that have excluded volume and weak attractive interactions with other spacers and stickers without any attraction preferences. ${ }^{15,16}$ This model has been effectively employed to study phase separating proteins, and how valency of stickers (specific interactions) affect the phase separation and aggregation. ${ }^{15}$ Most of these existing theoretical models, however, focus on the role of various interaction parameters on the equilibrium state of the interacting biopolymers. ${ }^{9,17-20}$ In contrast, the process of assembly and the role played by non-specific interactions in the formation of specific polymeric complexes is less clearly understood.

In this work, we study the role of non-specific interactions in driving the phase separation of polymers enabling the specifically interacting domains to form contacts in the 
polymer-dense phase. Using a coarse-grained representation of proteins, we explore various parameters - specific interaction strengths, non-specific interaction strengths, a fraction of non-specifically interacting residues and systems of different polymer types to understand their role in phase separation of biological macromolecules. The interacting proteins were modeled as semi-flexible polymer chains consisting of two domains, a small central patch involved in functional interactions (specific interactions) and other regions which participate in weak, promiscuous interactions (non-specific interactions). The results from off-lattice Langevin dynamics simulations show that non-specific assemblies could be an essential precursor to the formation of functional complexes. We systematically explore the parameter regimes where these functional domains find each other upon phase-separation. Our results suggest that non-specific interactions are essential in the formation of phase-separated states stabilized by specific interactions. We further tuned the fraction of non-specific interaction and its strength, which resulted in different assembling states. Using an extension of this model, we demonstrate how the transition between non-specific to specific interactiondominant assemblies is influenced by the presence of non-interacting polymer chains (crowders) and non-specifically interacting polymers in the system. Overall, our results shed light on how interactions involving noisy regions could be tuned to promote the formation of otherwise key, low-probability functional interactions. ${ }^{11,21,22}$

Table 1: Parameters abbreviations in simulations

\begin{tabular}{|l|l|}
\hline Parameters & Abbreviation \\
\hline$\epsilon_{\mathrm{ns}}$ & Non-specific interaction energy (pairwise) \\
\hline$\epsilon_{\mathrm{sp}}$ & Specific interaction energy (pairwise) \\
\hline $\mathrm{N}_{\mathrm{ns}}$ & Number of non-specific contacts per bead \\
\hline $\mathrm{N}_{\mathrm{sp}}$ & Number of specific contacts per bead \\
\hline $\mathrm{N}_{\mathrm{frac}}$ & Fraction of non-specifically interacting residues (given in percentage) \\
\hline $\mathrm{R}_{\mathrm{g}}^{\mathrm{red}}$ & Radius of gyration of red beads \\
\hline $\mathrm{N}^{\mathrm{lc}}$ & Number of polymers in largest cluster. \\
\hline $\mathrm{R}_{\mathrm{g}}^{\text {norm }}$ & Radius of gyration normalised $\left(\mathrm{R}_{\mathrm{g}}^{\text {red }} / \mathrm{N}^{\mathrm{lc}}\right)$ \\
\hline
\end{tabular}




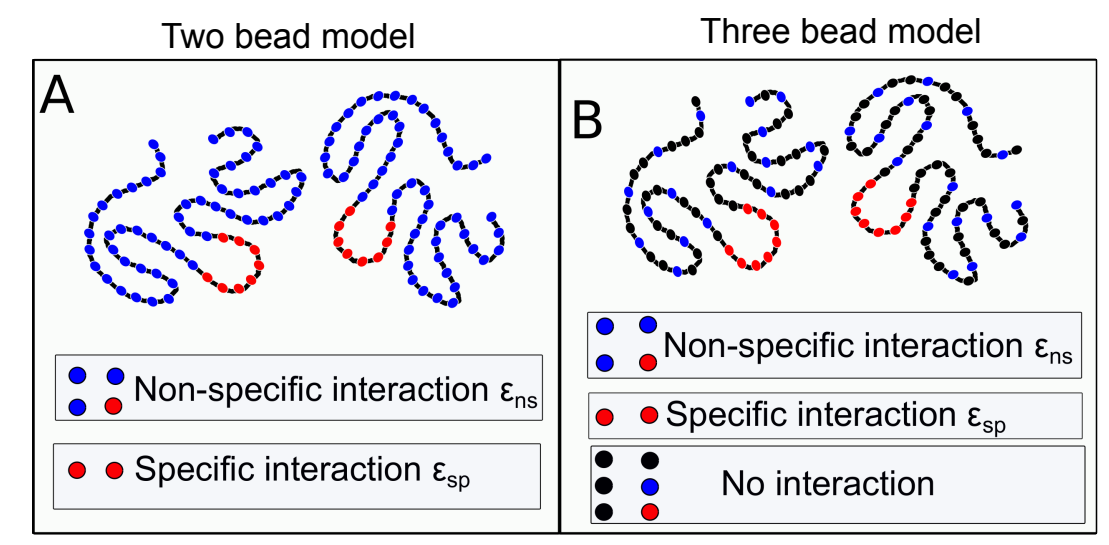

Figure 1: Bead-spring polymer model of protein. A) Chains consist of a central "functional" patch (red) surrounded by other beads which make up non-specifically interacting regions (blue). B) Three bead polymers with black beads representing non-interacting residues, in addition to specifically (red) and non-specifically (blue) interacting residues. Specific and non-specific interaction strengths are represented by $\epsilon_{\mathrm{sp}}$ and $\epsilon_{\mathrm{ns}}$, respectively, with $\epsilon_{\mathrm{sp}}>\epsilon_{\mathrm{ns}}$.

\section{Results}

As discussed in the model section, we model proteins as bead spring polymer chains, with each bead signifying an effective interaction site per region of one or a few amino acids. We simulate polymer chains that are 80-beads long and each having different types of interactions among the beads as shown in Fig. 1. Apart from the steric hindrance, the beads may have weak non-specific interaction with energy $\epsilon_{\mathrm{ns}}$, strong specific interaction with energy $\epsilon_{\mathrm{sp}}$ or no interaction as the case may be. We place multiple polymers inside a cubic box at random locations and compute their dynamics using Langevin dynamics and Replica Exchange Langevin Dynamics (RELD) simulations.

\section{Proteins as dynamic polymers: role of non-specific interaction in the formation of functional dimers}

Cellular function hinges on biomolecular recognition and the formation of specific proteinprotein complexes. Interestingly, interface residues involved in stabilizing biomolecular complexes account for only a tiny fraction of protein surfaces. Here, we first probe how the interplay between specific and non-specific interactions can shape the process of dimeriza- 
tion with coarse-grained polymer chains (Fig. 1A). We vary the $\epsilon_{\mathrm{ns}}$ and $\epsilon_{\mathrm{sp}}$ systematically and investigate the process of formation of mature dimers - a state where the two central red patches interact with each other. This mature dimeric state is similar to protein-protein complexes stabilized in the correct configurations that enable biomolecular function.
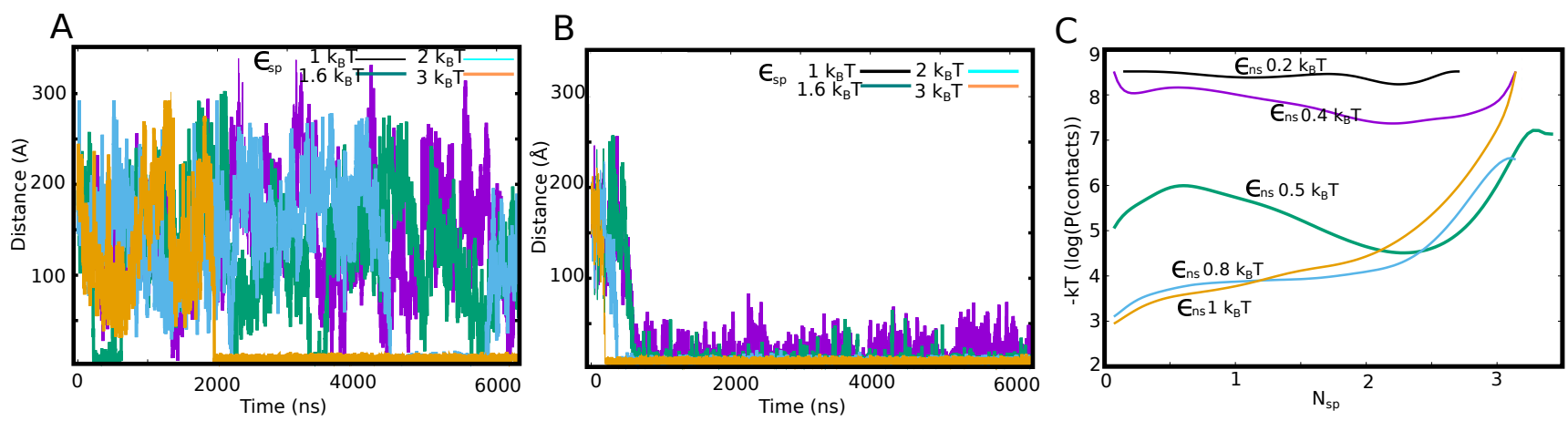

Figure 2: Distance between the center of masses of the two polymeric chains as a function of time. The strength of the $\epsilon_{\mathrm{ns}}$ in the two panels are A) $\left.0.2 \mathrm{k}_{\mathrm{B}} \mathrm{T}, \mathrm{B}\right) 0.6 \mathrm{k}_{\mathrm{B}} \mathrm{T}$. C) Free energy as a function of specific contacts per bead computed using replica exchange langevin dynamics simulations. Individual free energy curves represent simulations performed at different values of $\epsilon_{\mathrm{ns}}$. For an $\epsilon_{\mathrm{ns}}=0.5 \mathrm{k}_{\mathrm{B}} \mathrm{T}$ (green curve), there is a sharp minima for higher value of specific contacts per bead. For high and low values of $\epsilon_{\mathrm{ns}}$ this minima is not observed, suggesting that there is an optimal value of $\epsilon_{\mathrm{ns}}$ at which functional dimers are favored.

In Fig. 2A and Fig. 2B, we plot distance between center of masses of red patches in the two polymers, as a function of time, for varying $\epsilon_{\mathrm{ns}}$. At low non-specific interaction strengths $\epsilon_{\mathrm{ns}}=0.2 \mathrm{k}_{\mathrm{B}} \mathrm{T}$ in Fig. $2 \mathrm{~A}$ the two red patches form stable interactions only at extremely large values of specific interaction $\left(\epsilon_{\mathrm{sp}}=3 \mathrm{k}_{\mathrm{B}} \mathrm{T}\right.$, Orange curve). For values of $\epsilon_{\mathrm{sp}}$ lower than $3 \mathrm{k}_{\mathrm{B}} \mathrm{T}$, the two 'functional' patches do not meet or stabilize during the timescale of our simulation $(6 \mu \mathrm{s})$. At lower values of $\epsilon_{\mathrm{sp}}$, even when the red patches do come in contact with each other, they do not dwell long enough to make stable contacts as seen green curve of Fig. 2A, same observation is made for $\epsilon_{\mathrm{ns}}=0.4 \mathrm{k}_{\mathrm{B}} \mathrm{T}$ (see supplementary Fig. $1 \mathrm{~A}$ ). As we increase the strength of the $\epsilon_{\mathrm{ns}}$ to $0.6 \mathrm{k}_{\mathrm{B}} \mathrm{T}$ (Fig. 2B), the central patches form stable contacts at $\epsilon_{\mathrm{sp}}$ values as low as $1 \mathrm{k}_{\mathrm{B}} \mathrm{T}$. An increase in $\epsilon_{\mathrm{ns}}$ from $0.6 \mathrm{k}_{\mathrm{B}} \mathrm{T}$ to $1.0 \mathrm{k}_{\mathrm{B}} \mathrm{T}$ (supplementary Fig. 1B), results in a slowing down of time it takes for the specific interaction to form - this is due to stronger non-specific interaction (blue-blue) delaying the meeting of red-red patches. 
To further understand how $\epsilon_{\mathrm{ns}}$ could modulate both the equilibrium state as well as the kinetics of this transition, we performed parallel tempering (replica exchange) Langevin dynamics simulations to construct the free energy profiles for the dimeric system. We simulated 24 different replicas with fixed $\epsilon_{\mathrm{sp}}=1.2 \mathrm{k}_{\mathrm{B}} \mathrm{T}$, each spaced $4 \mathrm{~K}$ apart for a temperature range of 309-401 K. The free energy curves in Fig. 2C show that for very low values of $\epsilon_{\mathrm{ns}}$, we do not observe a clear minima at higher values of $\mathrm{N}_{\mathrm{sp}}$ (specific contacts per bead). Also, for very large values of $\epsilon_{\mathrm{ns}}$, we observe a steep barrier $\left(>3 \mathrm{k}_{\mathrm{B}} \mathrm{T}\right)$ to go from low to high values of specific contacts, suggesting that the system would prefer to remain in a non-specifically bound state. However, at an intermediate value of $\epsilon_{\mathrm{ns}}$, we see a defined minima for the higher value of $\mathrm{N}_{\mathrm{sp}}$, showing that the system would undergo a transition from the phase-separated non-specific dimeric state to the mature dimeric state in such a scenario. These results suggest that the meeting of functional interfaces (red patch) between two polymer molecules must be facilitated by weak $\epsilon_{\mathrm{ns}}$ that increases the dwelling time of a non-specifically bound dimer. The individual chains within the dimer can then undergo rearrangement, eventually resulting in mature dimers with the central specifically interacting patches in contact with each other.

\section{Functional biomolecular complexes: non-specific interaction, phase separation and maturation}

Phase-separated biological assemblies such as membrane-less organelles can often localize hundreds of molecules within them ${ }^{23-27}$ The concentrations of proteins within these clusters are several-fold higher than their bulk concentrations, facilitating favorable biomolecular interactions within the dense phase. Also, promiscuous interactions have been attributed to stabilizing the phase-separated state while allowing the specific interactions to form within the polymer-rich phase. In this context, we simulated 30 polymer chains, each having two patches with the same behavior as described so far (see Fig. 1B), in a box such that the concentration is $\approx 200 \mu \mathrm{M}$. We systematically probe the role of the non-functional regions 

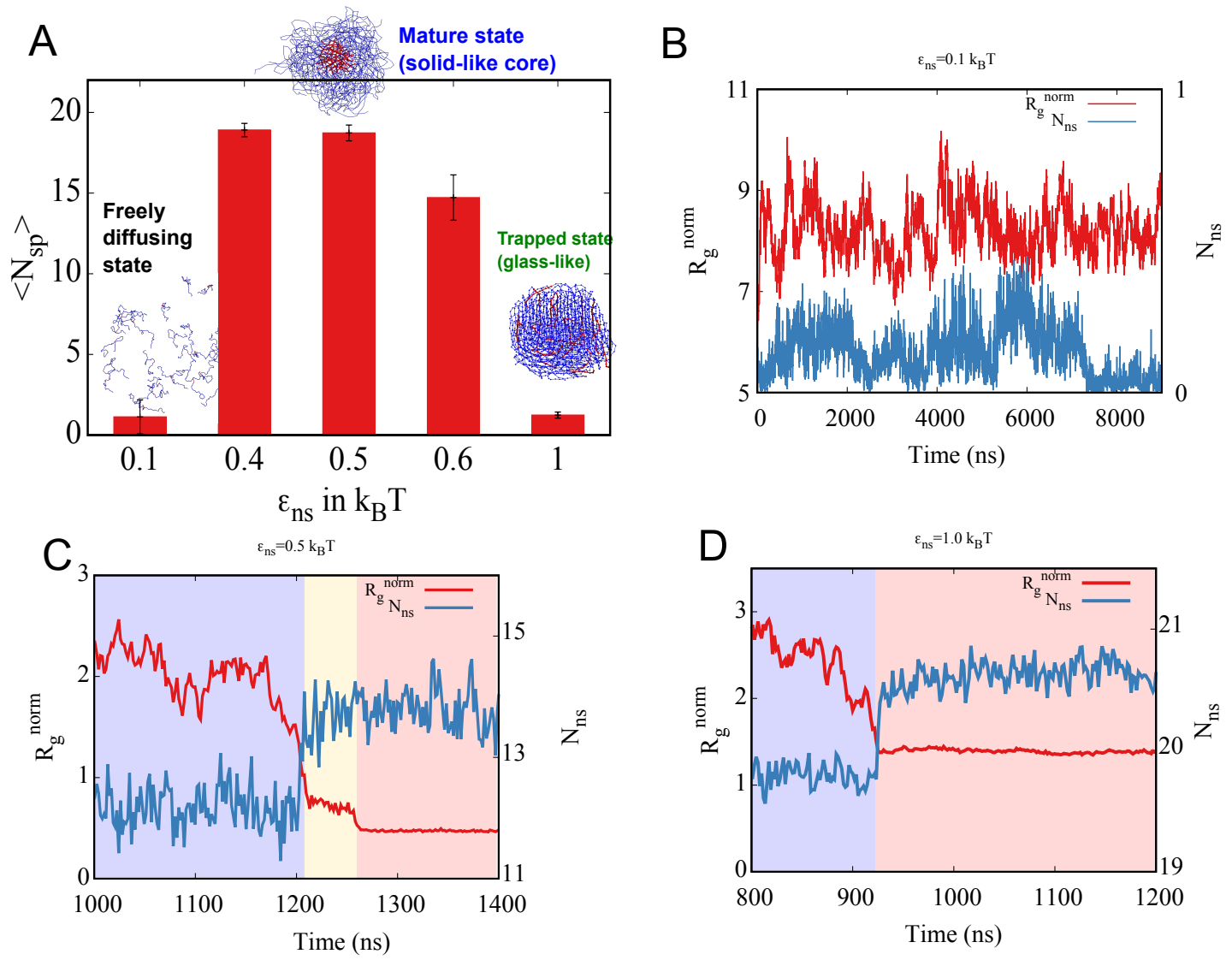

Figure 3: A) Average number of specific interaction per bead (red-red) $\mathrm{N}_{\mathrm{sp}}$ for different of non-specific interaction strength $\epsilon_{\mathrm{ns}}$ values. (B,C,D) Radius of gyration $\left(\mathrm{R}_{\mathrm{g}}^{\text {norm }}\right)$ and Number of non-specific interactions per bead $\left(\mathrm{N}_{\mathrm{ns}}\right)$ are plotted as a function of time for $\epsilon_{\mathrm{sp}}=1.2 \mathrm{k}_{\mathrm{B}} \mathrm{T}$. B) For weak $\epsilon_{\mathrm{ns}}=0.1 \mathrm{k}_{\mathrm{B}} \mathrm{T}$ we observe neglibile non-specific contacts in the system $\left(\mathrm{N}_{\mathrm{ns}} \approx 0\right)$. This is accompanied by large values of $\mathrm{R}_{\mathrm{g}}^{\text {norm }}$ suggesting that the stable multimers are not observed. C)For $\epsilon_{\mathrm{ns}}=0.5 \mathrm{k}_{\mathrm{B}} \mathrm{T}$, there is an initial regime of low $\mathrm{N}_{\mathrm{ns}}$ and large $\mathrm{R}_{\mathrm{g}}^{\text {norm }}$ while the polymer chains are yet to form multimeric clusters. This is followed by a regime (shaded yellow) where there is an increase in $\mathrm{N}_{\mathrm{ns}}$ suggesting the formation of multimers stabilized by non-specific interactions. This increase in $\mathrm{N}_{\mathrm{ns}}$ further enables the central functional patches to come in contact, indicated by the further decrease in $\mathrm{R}_{\mathrm{g}}^{\text {norm }}$ (red regime). A further increase in $\epsilon_{\mathrm{ns}}$ to $1 \mathrm{kT}$ results in the initial formation of non-specifically stabilized multimers without a further reorganization into a functional complex where the central red-patches do not phase-separate into a separate region. At these high values of $\epsilon_{\mathrm{ns}}$, the multimers are trapped in a glass-like state.

of the polymer chain in driving phase separation that enables the meeting of functional patches within the polymer-dense phase. For $\epsilon_{\mathrm{sp}}$ of $1.2 \mathrm{k}_{\mathrm{B}} \mathrm{T}$, there is negligible development of contacts between specific patches, at low values of $\epsilon_{\mathrm{ns}}$ of $0.1 \mathrm{k}_{\mathrm{B}} \mathrm{T}$ (Fig. 3A). As we increase the $\epsilon_{\mathrm{ns}}=0.1 \mathrm{k}_{\mathrm{B}} \mathrm{T}$ to $0.4 / 0.5 / 0.6 \mathrm{k}_{\mathrm{B}} \mathrm{T}$, we see a significant increase in the $\mathrm{N}_{\mathrm{sp}}$ resulting in 
a mature complex, where all red patches come together. However, as the strength of the non-specific interactions approaches that of the specific interactions $\left(\epsilon_{\mathrm{sp}}=1.2 \mathrm{k}_{\mathrm{B}} \mathrm{T}\right.$ and $\epsilon_{\mathrm{ns}}$ $\left.=1 \mathrm{k}_{\mathrm{B}} \mathrm{T}\right)$, there is a sudden decrease in the number of specific contacts suggesting a slow down in the search process of the specific interfaces. This suggests that despite the relatively higher strength of the interaction between the red patches, the ability to phase separate and form a mature multimer (solid-like core, see below) is modulated by the 'non-specific' regions of the polymer chain.

For a better understanding of the system, typical snapshots of polymer configurations from the simulation are shown near the graph (Fig. 3A). For low $\epsilon_{\mathrm{ns}}$ there are only a few $\mathrm{N}_{\mathrm{sp}}\left(\epsilon_{\mathrm{ns}}=0.1 \mathrm{k}_{\mathrm{B}} \mathrm{T}\right)$ and the corresponding polymer configuration shows that the polymers are scattered without much interaction as freely diffusing state. When the $\epsilon_{\mathrm{ns}}$ is increased to 0.4/0.5/0.6 $\mathrm{k}_{\mathrm{B}} \mathrm{T}$, all red patches from different polymers come together and form a mature complex with a solid-like core. The corresponding snapshot of the polymer configuration shows a central core of red beads forming a mature state with a solid-like core of red beads. It is interesting to note that the non-specific interaction leads to a phase separation-like phenomena further transitioned to a mature state. When the value of $\epsilon_{\mathrm{ns}}$ was increased further to $1.0 \mathrm{k}_{\mathrm{B}} \mathrm{T}$, we find decrease in the $\mathrm{N}_{\mathrm{sp}}$. The corresponding polymer configuration shows that even though the polymers are nearby, they are stuck with each other and have much less mobility; the state is like a kinetically trapped polymer in a "glass-like" state.

To further understand the kinetics of red patches finding each other with the help of non-specific blue beads, we tracked two parameters as a function of time: $\mathrm{N}_{\mathrm{ns}}$ (Number of non-specific contacts per bead) and $R_{\mathrm{g}}^{\text {norm }}$ (Radius of gyration of red beads, $R_{\mathrm{g}}^{\text {norm }}=\mathrm{R}_{\mathrm{g}}^{\mathrm{red}} / \mathrm{N}_{\mathrm{lc}}$ ) as in Fig. 3B,C\&D. For very weak non-specific interactions $\left(0.1 \mathrm{k}_{\mathrm{B}} \mathrm{T}\right.$, Fig. 3B) there is no development of non-specific contacts (blue curve) as represented in the $y_{2}$ axis (the Y-axis on the right side). At the same time, the $\mathrm{R}_{\mathrm{g}}^{\text {norm }}$ is very large (red curve, marked on the $y_{1}$ axis - the major Y-axis on the left side), suggesting that the red beads are far away and are not in contact. However, when $\epsilon_{\mathrm{ns}}=0.5 \mathrm{k}_{\mathrm{B}} \mathrm{T}$, the non-specific contacts per bead increases 

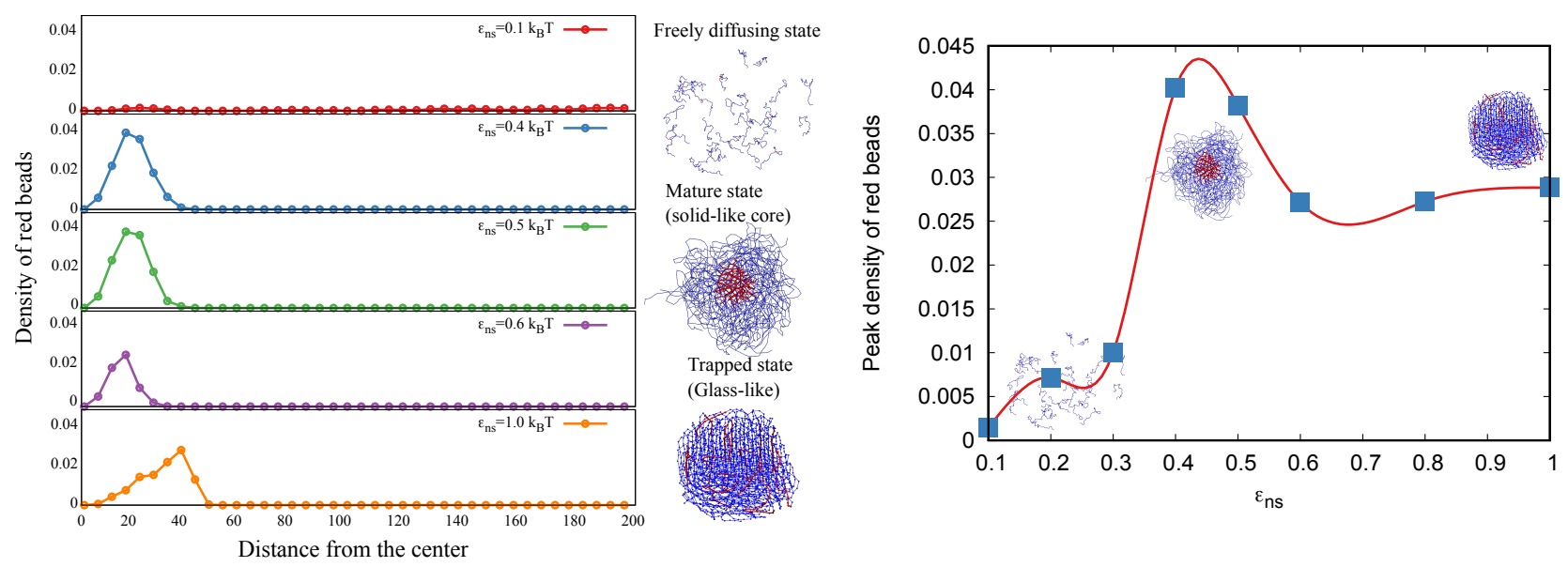

Figure 4: Density profiles. A) Density of red beads within a spherical shell with increasing radial distance from the center of a cluster. Individual panels represent density profiles for increasing values of $\epsilon_{n s}$ (top to bottom). B) Peak density of red beads within the single largest cluster shows an initial increase with $\epsilon_{\mathrm{ns}}$ followed by a decrease at large values of $\epsilon_{\mathrm{ns}}$.

substantially Fig. 3C (blue curve) as represented in the $y_{2}$ axis. At the same time, the redbeads start to come together and $\mathrm{R}_{\mathrm{g}}^{\text {norm }}$ decreases as in the blue region (Fig. 3C red curve represented on the $y_{1}$ axis). Even after the $\mathrm{N}_{\mathrm{ns}}$ saturate, the $\mathrm{R}_{\mathrm{g}}^{\text {norm }}$ keeps decreasing as in the yellow region; shows the kinetics of maturation to form a mature state with solid-like core from the non-specific phase-separated state. In Fig. $3 \mathrm{D}$, where $\epsilon_{\mathrm{ns}}=1.0 \mathrm{k}_{\mathrm{B}} \mathrm{T}, \mathrm{N}_{\mathrm{ns}}$ increase and $\mathrm{R}_{\mathrm{g}}^{\mathrm{norm}}$ decrease initially to form multimeric interaction as in blue region, then both the values saturate showing the formation of kinetically trapped polymers in a glass-like state (red region).

For different values of $\epsilon_{\mathrm{ns}}$, there are different plausible states for polymers to form. It can be in three different states, basically freely diffusing state, mature state with a solid-like core of red beads in the center, and glass-like trapped state, where the polymers form a multimer without red beads in the center. To differentiate these states of multimers formed, we plotted the number density of red beads from the center of the cluster towards its periphery (along radial direction) for various $\epsilon_{\mathrm{ns}}$ in Fig. $4 \mathrm{~A}$ and the peak density of the red beads in Fig. 4B. For $\epsilon_{\mathrm{ns}}=0.1 \mathrm{k}_{\mathrm{B}} \mathrm{T}$, the density is very low, suggesting polymers in a freely diffusing state. When $\epsilon_{\mathrm{ns}}=0.5 \mathrm{k}_{\mathrm{B}} \mathrm{T}$, the density is at its peak (as in Fig. 4B), indicating the red beads 
clustered at the center forming mature state with the solid-like core. For $\epsilon_{\mathrm{ns}}=1 \mathrm{k}_{\mathrm{B}} \mathrm{T}$ the density of the red beads is spread across the cluster, showing that the state in which polymers are kinetically trapped in a glass-like state (Fig. 4A).

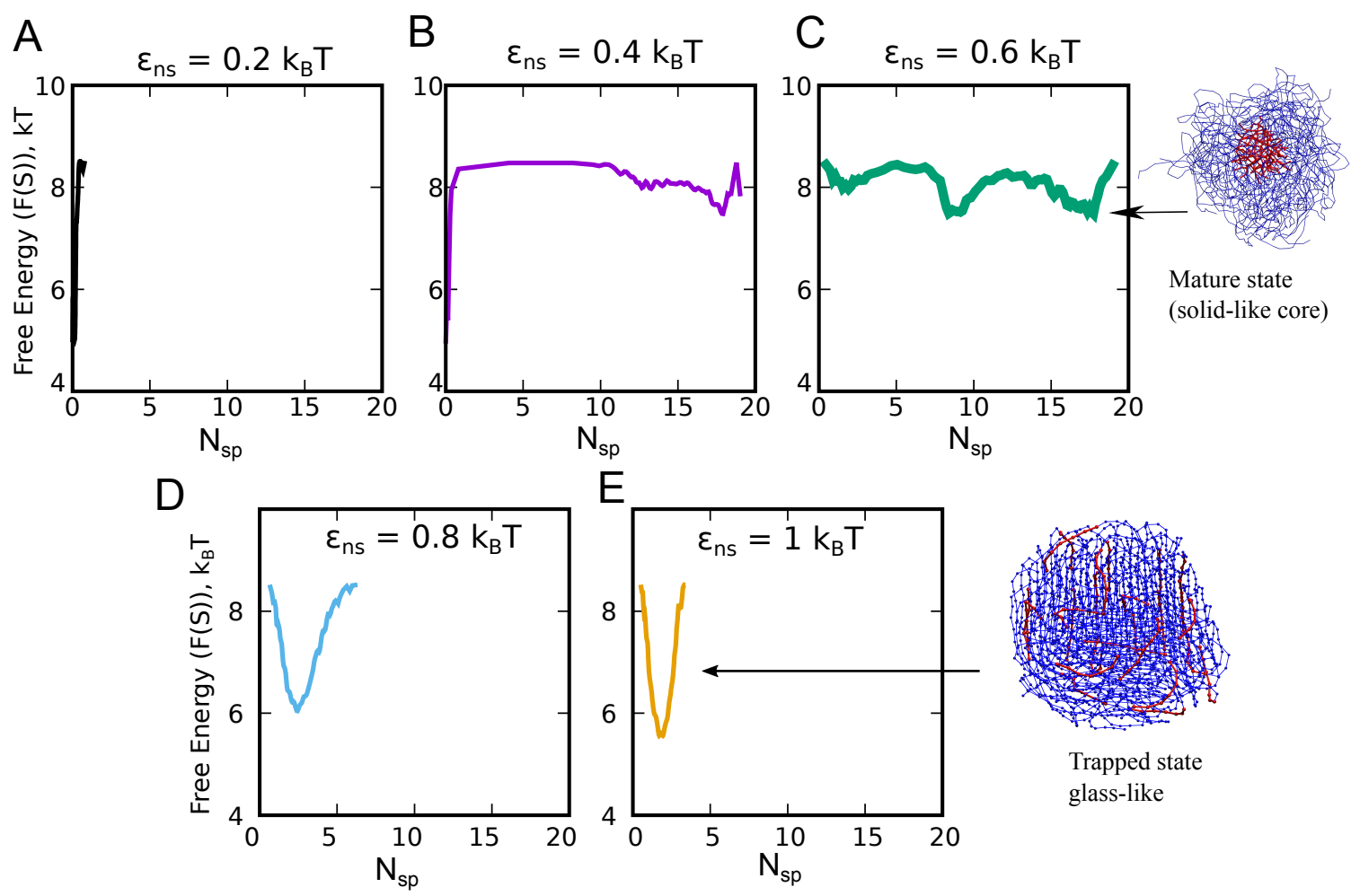

Figure 5: RELD simulations for the multimeric system. Free Energy profiles as a function of specific contacts as the order parameter. The free energy curves in A, B, C, D, E correspond to $\epsilon_{\mathrm{ns}}$ values of $0.2,0.4,0.6,0.8$ and $1 \mathrm{k}_{\mathrm{B}} \mathrm{T}$, respectively. For $\epsilon_{\mathrm{ns}}=0.2 \mathrm{k}_{\mathrm{B}} \mathrm{T}$, the system remains predominantly in a monomeric state. For an intermediate value of $\epsilon_{\mathrm{ns}}=0.4$ and $0.6 \mathrm{k}_{\mathrm{B}} \mathrm{T}$, we observe a sharp minima at higher values of specific contacts (per red bead), indicating that the system undergoes a transition to the mature state at this value of nonspecific interaction strength. For $\epsilon_{\mathrm{ns}}$ higher than $0.6 \mathrm{k}_{\mathrm{B}} \mathrm{T}$, the system remains trapped in the form of glass-like, non-specifically assembled aggregates, as evident by the sharp minima for low values of specific contacts.

To further establish the narrow range of $\epsilon_{\mathrm{ns}}$ in which the mature complex is favored, we also performed RELD simulations for the system with 30 polymeric chains with 24 replicas, with temperatures ranging from $309 \mathrm{~K}$ to $401 \mathrm{~K}$. We plot the resulting free energy profiles for the 30-mer system at a statistical temperature of $309 \mathrm{~K}$, with $\mathrm{N}_{\mathrm{sp}}$ as the order parameter used to identify the transition to the mature state with solid-like core (Fig. 5). Consistent with our RELD free energy profiles for the dimeric system, for weak $\epsilon_{\mathrm{ns}}$ in the multimeric 
system, we do not observe a defined minima at high values of $\mathrm{N}_{\mathrm{sp}}$. Also, for extremely strong $\epsilon_{\mathrm{ns}}$ (Fig. 5D and E), we observe a sharp free energy minima for low values of specific contacts, suggesting that the system remains a kinetically trapped glass-like state and does not access the mature state in such a scenario. Interestingly, for an intermediate value of $\epsilon_{\mathrm{ns}}=0.4$ and $0.6 \mathrm{k}_{\mathrm{B}} \mathrm{T}$, we observe a defined minima for large specific contact numbers establishing that the mature state gets facilitated in such a regime of $\epsilon_{\mathrm{ns}}$ (see Fig. 5B and C). Overall, these results suggest that the phase separation of protein and mature state (solid-like core) is favoured in a very narrow regime of $\epsilon_{\mathrm{ns}}$, and a deviation to either side would result in dramatically different equilibrium and dynamic properties.

Table 2: Heterogeneous mix of polymer definition and interaction involved in them and number of polymers in the system.

\begin{tabular}{|l|l|l|}
\hline $\begin{array}{l}\text { System } \\
\text { names }\end{array}$ & Different types of polymers in simulation box & $\begin{array}{l}\text { Number of } \\
\text { polymers }\end{array}$ \\
\hline Default & $\begin{array}{l}\text { type } \mathbf{1} \text { polymer with blue and red beads having the follow- } \\
\text { ing interactions. Blue-blue: } \epsilon_{\mathrm{ns}}=0.5 \mathrm{k}_{\mathrm{B}} \mathrm{T} \text {; blue-red: red-red: } \\
\epsilon_{\mathrm{sp}}=1.2 \mathrm{k}_{\mathrm{B}} \mathrm{T}\end{array}$ & 30 of type 1 \\
\hline System $\mathbf{1}$ & $\begin{array}{l}\text { beads having following interactions black-black: no interac- } \\
\text { tion, black red: no interaction, red-red: } \epsilon_{\mathrm{sp}}=1.2 \mathrm{k}_{\mathrm{B}} \mathrm{T}, \text { blue- }\end{array}$ & $\begin{array}{l}15 \text { of type } 1 \& \\
\text { black: no interaction }\end{array}$ \\
\hline System 2 2 & $\begin{array}{l}\text { type } \mathbf{1} \text { as above; type } \mathbf{3} \text { polymer with green beads and red } \\
\text { beads with following interactions green-green: } \epsilon_{\mathrm{ns}}=0.5 \mathrm{k}_{\mathrm{B}} \mathrm{T}, \\
\text { green-red: } \epsilon_{\mathrm{ns}}=0.5 \mathrm{k}_{\mathrm{B}} \mathrm{T}, \text { red-red: } \epsilon_{\mathrm{sp}}=1.2 \mathrm{k}_{\mathrm{B}} \mathrm{T} \text { and green- } \\
\text { blue: no interaction. }\end{array}$ & $\begin{array}{l}15 \text { of type } 1 \& \\
15 \text { of type } 3 .\end{array}$ \\
\hline
\end{tabular}

\section{Heterogeneous mix of polymers: Phase separation and dynamics}

So far, we have simulated multiple copies of the same kind of polymer (two bead polymer model Fig.1). The simulation suggests that critical strength of non-specific interaction is required for the formation of multimer and their subsequent maturation. In this section, we simulate a heterogeneous system composed of a mix of polymer chains and investigate their assembly into multimer for various scenarios described here. 


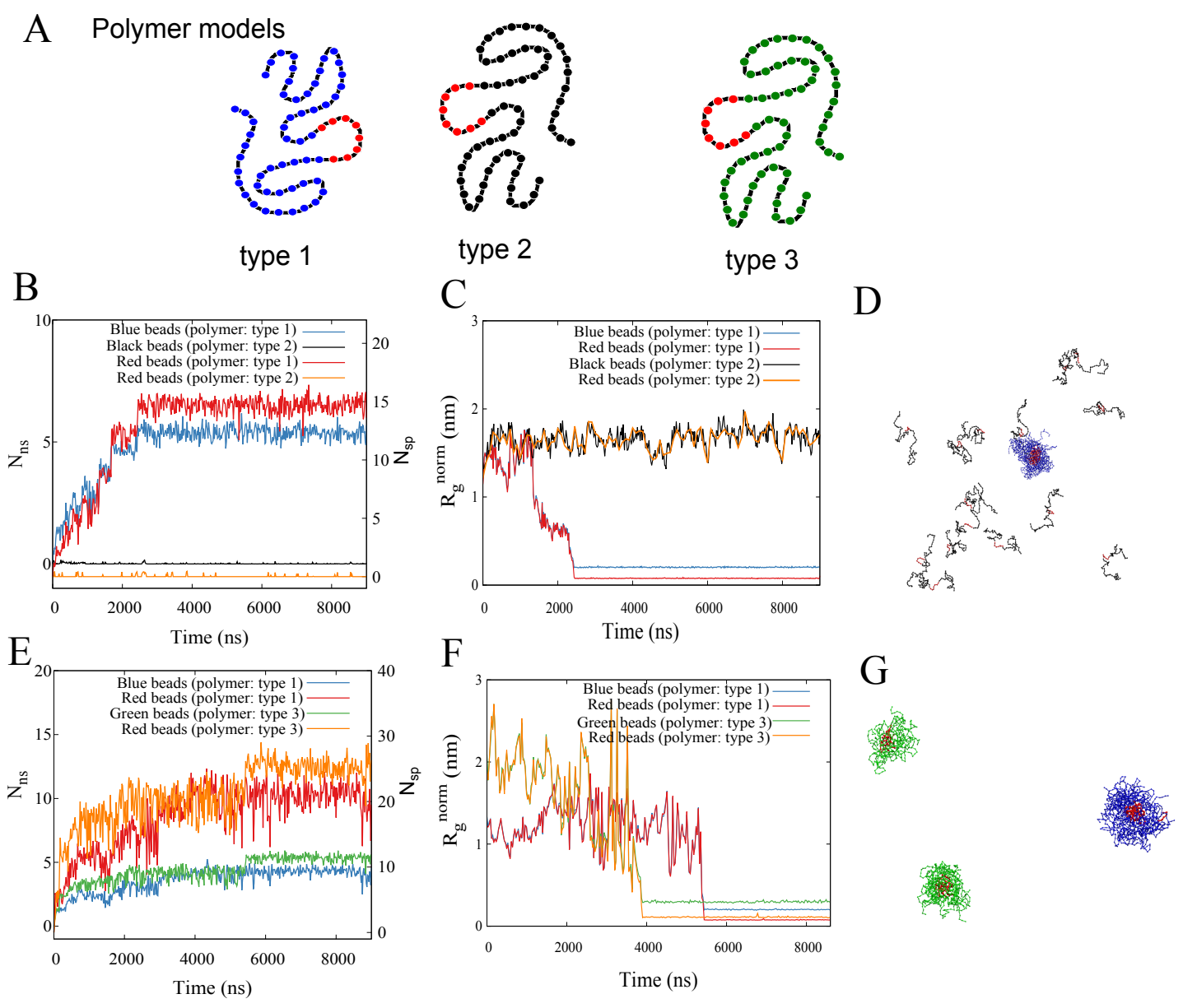

Figure 6: A) Polymer model: Cartoon of the Initial configuration of simulation, polymer type1 - blue-red polymer with blue beads non-specifically interacting and red beads specifically interacting, type $\mathbf{2}$ - black-red polymer, where black beads are non-interacting beads and red beads are specifically interacting, type $\mathbf{3}$ - green-red polymer with non-specifically interacting green beads and specifically interacting red beads. B) Non-specific contacts per bead ( $\mathrm{N}_{\mathrm{ns}}$ in y1 axis- major y-axis) and specific contacts per bead ( $N_{s p}$, y2 axis) are plotted over time for different types of beads from two polymers from System 1. C) Radius of gyration $\mathrm{R}_{\mathrm{g}}^{\text {norm }}$ of different beads from two different polymers is plotted over time from System 1 . D) Snapshot of the final trajectory from the simulation showing mature multimer formed by blue-red bead polymer and black-red polymer - freely diffusing in System 1. E) Non-specific contacts per bead $\left(\mathrm{N}_{\mathrm{ns}}\right.$ in y1 axis- major y-axis) and specific contacts per bead $\left(N_{s p}\right.$, y2 axis) are plotted over time for different types of beads from two polymers from System 2. F) Radius of gyration $\mathrm{R}_{\mathrm{g}}^{\text {norm }}$ of different beads from two different polymers is plotted over time from System 2. G) Snapshot of the final trajectory from the simulation shows the mature multimers formed in System 2.

In the first scenario, we simulated two types of polymers described as System 1 in Table 2. When these two different types of polymers are mixed and simulated, the question is 
whether we will see the polymers with interacting beads (type 1) mixed between the polymers with non-interacting beads (type 2) or complete phase separation between interacting and non-interacting beads consisting of polymers. In Fig. 6B non-specific contacts per bead (as $\mathrm{N}_{\mathrm{sp}}$ in $\mathrm{y} 1$-axis) for blue beads from type 1 and black beads from type 2 polymer and specific contacts per bead (as $\mathrm{N}_{\mathrm{sp}}$ in y2-axis) for red beads in both polymers are plotted over time. There is a steady increase in contacts per bead (both $\mathrm{N}_{\mathrm{ns}}$ and $\mathrm{N}_{\mathrm{sp}}$ ) in type 1 polymer, while no contacts are formed in type 2 polymer. In Fig. 6C, the radius of gyration $\left(\mathrm{R}_{\mathrm{g}}^{\mathrm{norm}}\right)$ for each group of beads is plotted over time; for beads in type 1 polymer, $\mathrm{R}_{\mathrm{g}}^{\mathrm{norm}}$ is decreasing as a function of time, suggesting that these polymers are developing contacts. In comparison, the type 2 polymer beads (black and yellow curve Fig. $6 \mathrm{~B}$ and $\mathrm{C}$ ) have high $\mathrm{R}_{\mathrm{g}}^{\text {norm }}$ and low contacts, while type 1 polymer beads (blue and red curve Fig. 6B and C) have low $\mathrm{R}_{\mathrm{g}}^{\text {norm }}$ and high contacts; Red beads in type 1 polymer have even higher contacts and low $\mathrm{R}_{\mathrm{g}}^{\text {norm }}$ than the blue beads suggesting the formation of mature multimer with a solid-like core by polymer of type 1 . This can also be seen from a representative snapshot of a late time configuration from the simulation (see Fig. 6D). In summary, the polymers of two different types are phase separating even though they have a common red core for attracting. This suggests the importance of weak $\epsilon_{\mathrm{ns}}$ in polymer self-assembly. Note that the type 2 polymer not having weak $\epsilon_{\mathrm{ns}}$ are randomly diffusing.

Given that the non-specific interaction is crucial for the self-assembly of polymers, the next question would be the polymers type 1 and 3 from System 2 forms multimer (See Table 2). Essentially both polymers contain the same types of beads, but blue beads from type 1 and green beads from type 3 do not attract each other and have steric repulsion. $\mathrm{N}_{\mathrm{ns}}$ for green and blue beads (y1 axis, Fig. 6E) along with $\mathrm{N}_{\mathrm{sp}}$ of red beads (y2 axis, Fig. 6E) in polymer type 1 and type 3 are plotted, and the contacts for all the bead types from both polymers increase with time and equilibrate. While radius of gyration $\left(\mathrm{R}_{\mathrm{g}}^{\text {norm }}\right)$ of the beads plotted in Fig. 6F decreases for all the types of beads from different polymers, showing the formation of the multimer. High contacts per bead and low $\mathrm{R}_{\mathrm{g}}^{\text {norm }}$ for red beads from both polymers 
show the formation of mature multimer in both the polymer types. In the snapshot of the late time configuration of simulation (Fig. 6G), mature multimers of two types of polymers are observed. The disliking nature of blue and green beads from respective polymers makes these distinct multimeric phases emerge.

These simulations are examples of the various types of multimer formation and phase separation, a phenomenon that can be achieved in biology by tuning the parameters of non-specifically interacting beads and the interacting partners.

\section{Three bead polymer model: non-specific regions drive mature mul- timers in a very narrow parameter regime}

The simulations so far dealt with the self-organization of a homogeneous system of polymer chains made up of two kinds of beads. The results demonstrate how the interactions between the non-specific region can act as a handle for tuning the timescale of multimer formation and phase separation-like behavior of biopolymers. However, the molecular language of proteins composed of 20 different building blocks (amino acids) with different interaction propensities enables a more subtle tuning of the self-assembly/phase-separation phenomenon. We extend our two-bead model to three bead polymer model with a fraction of non-interacting beads. As in the current model, the polymer has a central patch of red beads having high specific interaction energy and a fraction of the remaining beads has non-specific interaction, and other beads are completely non-interacting (only steric effects) as shown Fig. 1C. This allows us to systematically study the interplay between the strength and concentration of nonspecific interaction.

In this section, we demonstrate the amenability of the self-assembly/phase-separation landscape to be fine-tuned in a scenario where the $\epsilon_{\mathrm{ns}}$ is dispersed across the non-functional regions of the polymer chain. In Fig. 7E and F, we monitor the self-organization of multiple 80-bead long hetero-polymer chains. For a fixed value of interaction strength of $\epsilon_{\mathrm{sp}}=1.2$ $\mathrm{k}_{\mathrm{B}} \mathrm{T}$ between the core residues, we systematically vary the strength of non-specific interac- 


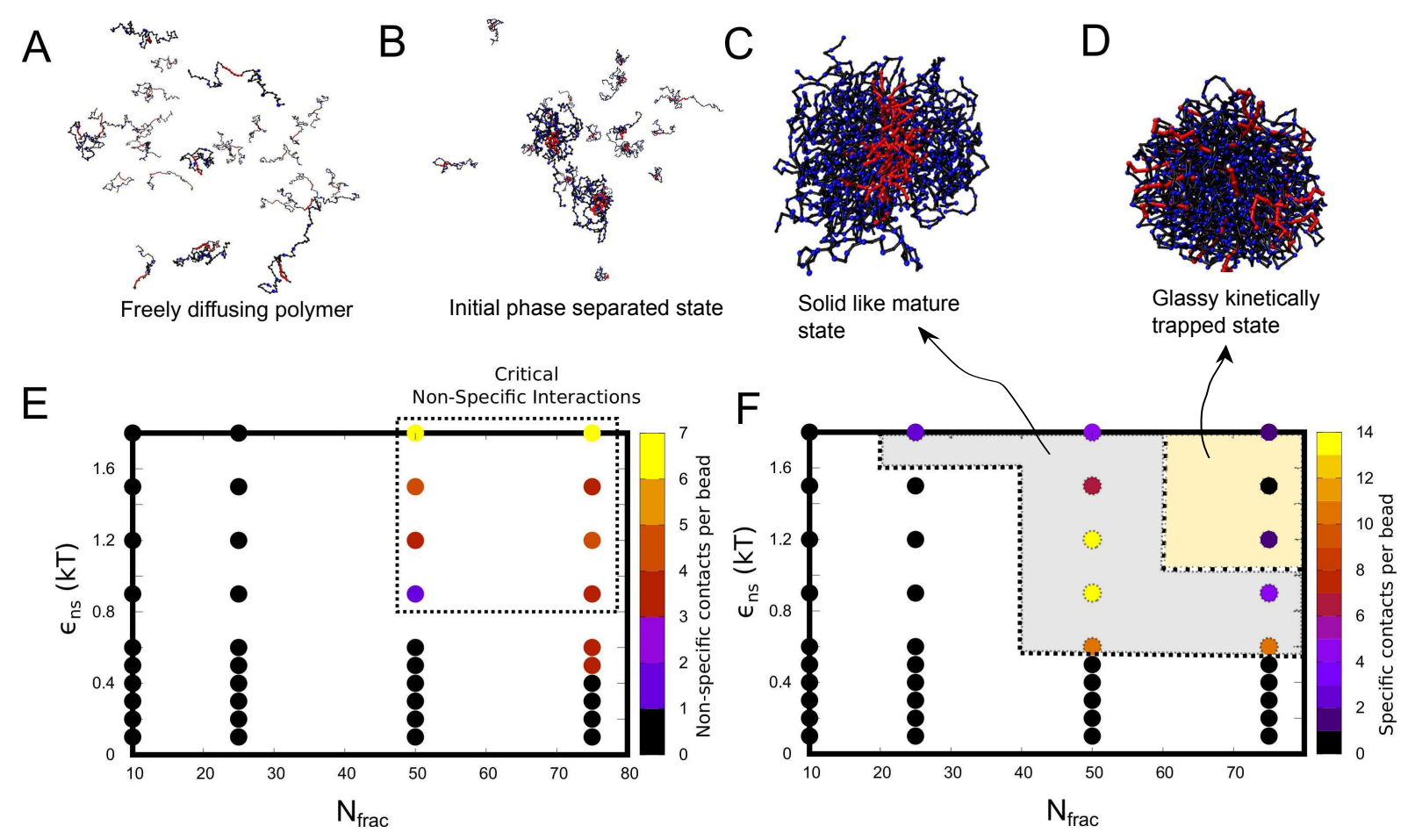

Figure 7: A) Snapshots of A) freely diffusing polymer in the simulation. B) early nonspecifically bound initial multimer state, C) mature multimer and D) glass-like, kinetically trapped multimeric state for a 3-bead polymeric system. E) Non-specific contacts , $\mathrm{N}_{\mathrm{ns}}$, per bead is plotted for varying non-specific interaction $\epsilon_{\mathrm{ns}}$ and fraction of non-specifically interacting beads $\left(\mathrm{N}_{\text {frac }}\right.$, given in percentage). The dotted box indicate the critical values of non-specific interaction and $\mathrm{N}_{\text {frac }}$-fraction of non-specific beads required for formation mature multimer complex. F) Specific contacts, $\mathrm{N}_{\mathrm{sp}}$, as a function of varying non-specific interaction, $\epsilon_{\mathrm{ns}}$, and fraction of non-specifically interacting beads. The grey area covered inside dotted line is the region where the formation of multimer leading to mature complex is plausible. The simulations were performed for a fixed specific interaction strength, $\epsilon_{\mathrm{sp}}$, of $1.2 \mathrm{k}_{\mathrm{B}} \mathrm{T}$.

tion $\epsilon_{\mathrm{ns}}$ and fraction of non-specifically interacting residues $\left(\mathrm{N}_{\text {frac }}\right)$ to probe the parameter regime, wherein functional complex formation (red-red beads interaction or specific contacts formation) could occur.

At very low fraction of non-specifically interacting residues $\mathrm{N}_{\text {frac }}$, the polymers are not involved in non-specific contacts (Fig. 7E, black dots). In this parameter regime of $\epsilon_{\mathrm{ns}}$ and $\mathrm{N}_{\text {frac }}$, the core regions of these chains do not form mature complexes suggesting that polymers would rather be freely diffusing state (see a snapshot of simulation in Fig. 7A) and the crucial role played by the non-functional residues (with $\epsilon_{\mathrm{ns}}$ ) information of functional assemblies. In 
the regime of high $\epsilon_{\mathrm{ns}}\left(2 \mathrm{k}_{\mathrm{B}} \mathrm{T}\right)$ with a low $\mathrm{N}_{\text {frac }}(25 \%)$ and low $\epsilon_{\mathrm{ns}}\left(0.6 \mathrm{k}_{\mathrm{B}} \mathrm{T}\right)$ with a high $\mathrm{N}_{\text {frac }}$ $(75 \%)$, there will be few clusters of multimers in the system as shown in the Fig. 7B, due to random interaction of polymers with cumulatively high interactions present. Further, in the parameter regime demarcated by the dotted boxed region in Fig. 7E wherein non-specific contacts start appearing, the polymer assemblies also form functional contacts (Fig. 7F, grey shaded region), suggesting polymers would have formed a mature multimer (see a snapshot of simulation in Fig. 7C). However, for extreme values of $\epsilon_{\mathrm{ns}}$ and $\mathrm{N}_{\text {frac }}$, there is a reduction in functional contacts (Fig. 7F, yellow shaded region) and the system would rather be in a trapped state as shown in Fig. 7D. This is because high non-specific interaction in many beads $(75 \%)$ makes them interact more than the specific beads. These results corroborate the two-bead model's findings wherein a critical non-specific interaction strength is vital for self-assembly en route to maturation. Also, very few interactions between the residues of the non-core region can drive the formation of critical interactions between the core regions. In such a regime with a few interaction sites and a narrow parameter range for self-assembly, subtle modifications such as phosphorylation of these non-core residues could significantly alter the phase behavior, critical concentrations, and dynamicity of the condensates. ${ }^{18,28-30}$ Conversely, few mutations resulting in extremely 'sticky' non-specific regions might result in loss of the ability of the polymers to form functional complexes.

\section{Discussion and Conclusion}

Biological reactions rely heavily on specificity and the ability of biomolecules to not only bind to the correct binding partners but also assume mature interaction configurations. However, the concentration of biomolecules like proteins is typically very low $(\approx n M)$ within cells, making the reactions malleable to tuning by external factors. The cellular environment is crowded with different types of biomolecules diffusing and promiscuously interacting with each other. ${ }^{9}$ Large stretches within protein sequences are not involved in the protein-protein interactions. 
This leads to an interesting question: can regions within proteins that are not involved in mature functional interfaces influence the process of formation of functional protein-protein complexes? At biologically relevant concentrations, could these non-interfacial residues have co-evolved to tune the binding affinities and dissociation constants of these proteins? How does the interplay between specific and promiscuous interactions shape the fate of these complexes?

Given the sheer size of the proteome and the vast number of protein-protein interactions that underlie cellular processes, phenomenological models could help us shed light on the physical mechanisms underlying the process of the formation of mature complexes. ${ }^{25,31,32}$ Previous theoretical studies have attempted to understand the effect of promiscuous interactions on the ability of proteins to bind specifically to their partners. ${ }^{19}$ However, there is a void in bottom-up studies elucidating the potential role of promiscuous interactions in promoting the formation of mature protein-protein complexes. To study this, we employed coarse-grained semi-flexible polymer chains and simulated them using Langevin dynamics and replica-exchange Langevin dynamics. The summary of our findings are as follows: (1) simulating dimer dynamics, we find that a narrow window of non-specific interaction strengths leads to a quick meeting of functional patches and formation of stable dimer complex (Fig. 2). (2) We show that the narrow optimal range of non-specific interaction strength also affects the formation of functional complexes with multiple polymers (a mature state with solid-like core) (Fig. 3). (3) Using RELD, we show that the optimal parameter regime leads to a free energy landscape having minima corresponding to the matured functional state of polymeric assemblies with specific interactions stabilising the assemblies (Fig. 5). (4) We then examined a heterogeneous mix of different types of polymers and showed that this leads to different kinds of complexes depending on the nature of interactions (Fig. 6). (5) We also simulated polymers varying the fraction of non-specifically interacting beads and their strengths and showed that there is a rich phase diagram emerging; the phase diagram suggests how assembly states of polymers might be altered (by going from one phase to the 
other) in realistic situations (Fig. 7).

Various interactome studies suggest that non-interacting or noisy regions make up a large fraction of the proteome. ${ }^{13}$ Further, several RNA bindings proteins are known to harbor intrinsically disordered regions, which facilitate RNP-granule assembly. ${ }^{33,34}$ The spacer-sticker model for proteins has been previously employed to study the role of physical parameters such as specific interaction valencies and strengths in shaping equilibrium aspects of phase separation. ${ }^{15,35}$ In these models, the stickers are considered to be the primary drivers of phase separation. This raises an interesting question - could the spacer regions play a role in shaping the kinetics of phase separation? In other words, can the non-specific interactions involving spacer regions influence inter-sticker interactions? This question gains importance in the context of membrane-less organelles, which facilitate the formation of inter-protein contacts within a high-density condensate phase.

Our simulations point towards a potential role of the non-interacting surfaces in facilitating the formation of mature protein-protein complexes. The current study provides a mechanistic basis for the narrow range of non-specific interaction strengths that promote protein-protein interactions. For the sake of simplicity, we modeled proteins as semi-flexible polymers with no defined native configuration. This makes it computationally tractable and allows us to sample a wider parameter space. The role of non-specific interactions in promoting the maturation of protein complexes with defined structural folds and lower conformational flexibility is to be further probed. Also, our simulations were either performed with one type or two types of polymers. In future, the simulations can be extended to multiple types of polymers in a system.

\section{Methods}

Despite the growing body of experimental literature on biomolecular phase separation, the multi-component nature of the problem makes it hard to unravel experimentally. Hence, 
Molecular dynamics simulations and computational models are very valuable and have been traditionally used to understand various aspects of biomolecular structural dynamics. ${ }^{36}$ However, the scale of this problem makes it hard to probe systematically using conventional atomistic simulations. The widespread instances of the phenomenon mean that it can be treated as a problem of self-assembling polymer chains. To understand the contribution of noisy interactions in driving the formation of mature polymer complexes, we developed a semi-flexible polymer model. ${ }^{37-41}$ In order to study the process of macromolecular chains finding each other, we performed Langevin dynamics simulations. In our study, we consider $\mathrm{N}$ flexible polymer chains, each made up of ' $\mathrm{X}$ ' red beads and ' $\mathrm{Y}$ ' blue beads, in a cubic box with periodic boundary conditions. The red beads represent residues involved in native functional interactions, while blue beads represent residues with the non-functional interaction Fig. 1.

Polymer chains of length 'M' beads are bonded via the harmonic potential with energy,

$$
\mathrm{E}_{\text {stretching }}=\mathrm{k}_{\mathrm{s}} \sum_{\mathrm{i}=1}^{\mathrm{M}-1}\left(\left|\overrightarrow{\mathrm{r}}_{\mathrm{i}}-\overrightarrow{\mathrm{r}}_{\mathrm{i}+1}\right|-\mathrm{r}_{0}\right)^{2}
$$

where $\overrightarrow{\mathrm{r}}_{\mathrm{i}}$ and $\vec{r}_{i+1}$ refer to $i^{\text {th }}$ and $(i+1)^{\text {th }}$ bead positions, respectively; $r_{0}$ refers to the equilibrium bond length and $k_{s}$ represents the spring constant. This interaction ensures the connectivity between the beads of a polymer chain. To model bending rigidity, any two neighboring bonds in a polymer interact via the following potential

$$
\mathrm{E}_{\text {bending }}=\kappa \sum_{\mathrm{i}=1}^{\mathrm{M}-2}\left(1-\cos \theta_{\mathrm{i}}\right)
$$

where $\theta_{i}$ refers to the angle between $i^{t h}$ and $(i+1)^{t h}$ bond, and $\kappa$ is the bending stiffness. All other non-bonded, inter-bead interactions were modeled using the Lennard-Jones (LJ) potential,

$$
\mathrm{E}_{\mathrm{nb}}=4 \epsilon \sum_{\mathrm{i}<\mathrm{j}}\left[\left(\frac{\sigma}{\left|\overrightarrow{\mathrm{r}_{\mathrm{i}}}-\overrightarrow{\mathrm{r}_{\mathrm{j}}}\right|}\right)^{12}-\left(\frac{\sigma}{\left|\overrightarrow{\mathrm{r}_{\mathrm{i}}}-\overrightarrow{\mathrm{r}_{\mathrm{j}}}\right|}\right)^{6}\right]
$$


for all $\left|\overrightarrow{r_{i}}-\overrightarrow{r_{j}}\right|<r_{c}$, where $r_{c}$ refers to the cutoff distance beyond which the non-bonded potentials are neglected. The cutoff for the LJ calculations was kept at 2.5 times of $\sigma$. Note that this function has two parts: a part that models steric repulsion when the beads overlap and an attractive part otherwise. For ease of implementation, in this model, this attractive part is used as an effective interaction that accounts for all attractive short-range forces. $\epsilon$ signifies the strength of the attractive interaction and has the units of energy. In our study, we choose different values of $\epsilon$ for interactions between red-red beads, as compared to those involving the blue beads. Since the interactions involving the blue beads model weak, nonspecific contacts, they are lower in magnitude than the red-red interactions, which describe mature, native contacts. We systematically vary these two interactions to understand their role in driving the formation of mature complexes, keeping $\epsilon_{\mathrm{sp}}>\epsilon_{\mathrm{ns}}$. Note that the bending energy parameter $(\kappa)$ controls the stiffness of the individual polymer chains, while the LJ potential parameter $(\epsilon)$ controls the interaction between any two beads (inter-chain and intra-chain).

Simulation parameters: In our study, we used the LAMMPS molecular dynamics package to perform the dynamic simulations, ${ }^{42}$ where the simulator solves Newton's equations with viscous force and a Langevin thermostat, ensuring an NVT ensemble with temperature $\mathrm{T}=310 \mathrm{~K}$. An integration timestep $(d t)$ of $30 \mathrm{fs}$ was used for the simulations. Polymer chains of length $\mathrm{M}=80$ beads and mass of each bead was considered to be 110 Da (average mass of amino acids). The size parameter for the beads is taken as $\sigma=4.5 \AA$. The parameters for the bonded springs was fixed as $\mathrm{r}_{0}=4.5 \AA$ and $\mathrm{k}_{\mathrm{s}}=10 \mathrm{kcal} / \mathrm{mol}$. The damping time for the Langevin thermostat was 1.2 ps. Similar values for these parameters have been previously used to perform coarse-grained protein simulations by Bellesia et al. and Bieler et al. ${ }^{43,44}$

Replica Exchange Langevin Dynamics: In order to calculate the free energy profiles for the dimeric and multi-polymer systems (Fig. 2C and Fig. 5), we employed the parallel tempering Langevin dynamics technique. In this approach, 24 independent, non-interacting copies (replicas), each at different temperatures between $309 \mathrm{~K}$ - $401 \mathrm{~K}$ each $4 \mathrm{~K}$ apart, were 
simulated under the NVT ensemble. In order to ensure proper sampling of equilibrium states, an exchange of configurations between different replicas is attempted every 0.5 ns. In this algorithm, a successful swap of configurations between replicas (at different temperatures) occurs based on the Boltzmann probability. ${ }^{45,46}$ Each of the individual replicas was simulated for a timescale of $9 \mu \mathrm{s}$. Using the combination of conventional Molecular dynamics and the Monte Carlo algorithm, this technique has been commonly used to enable efficient sampling of the conformational space for problems such as protein folding and self-assembly.

\section{Acknowledgements}

The authors would like to thank Prof. B.J. Rao, Prof. Dibyendu Das and Prof. M.Muthukumar for their suggestions and discussions related to the work.

\section{References}

(1) Boeynaems, S.; Alberti, S.; Fawzi, N. L.; Mittag, T.; Polymenidou, M.; Rousseau, F.; Schymkowitz, J.; Shorter, J.; Wolozin, B.; Van Den Bosch, L.; Tompa, P.; Fuxreiter, M. Protein Phase Separation: A New Phase in Cell Biology. Trends in Cell Biology 2018, 28, 420-435.

(2) Caragine, C. M.; Haley, S. C.; Zidovska, A. Nucleolar dynamics and interactions with nucleoplasm in living cells. 2019, 1-21.

(3) Saha, S.; Weber, C. A.; Nousch, M.; Adame-Arana, O.; Hoege, C.; Hein, M. Y.; Osborne-Nishimura, E.; Mahamid, J.; Jahnel, M.; Jawerth, L.; Pozniakovski, A.; Eckmann, C. R.; Jülicher, F.; Hyman, A. A. Polar Positioning of Phase-Separated Liquid Compartments in Cells Regulated by an mRNA Competition Mechanism. Cell 2016, 166, 1572-1584.e16. 
(4) Bergeron-Sandoval, L.-P.; Safaee, N.; Michnick, S. Mechanisms and Consequences of Macromolecular Phase Separation. Cell 2016, 165, 1067 - 1079.

(5) Landry, C.; Levy, E.; Rabbo, D. A.; Tarassov, K.; Michnick, S. Extracting Insight from Noisy Cellular Networks. Cell 2013, 155, 983 - 989.

(6) Landry, C. R.; Levy, E. D.; Michnick, S. W. Weak functional constraints on phosphoproteomes. Trends in Genetics 2009, 25, 193 - 197.

(7) Levy, E. D.; Michnick, S. W.; Landry, C. R. Protein abundance is key to distinguish promiscuous from functional phosphorylation based on evolutionary information. Philosophical Transactions of the Royal Society B: Biological Sciences 2012, 367, 2594-2606.

(8) Tarassov, K.; Messier, V.; Landry, C. R.; Radinovic, S.; Molina, M. M. S.; Shames, I.; Malitskaya, Y.; Vogel, J.; Bussey, H.; Michnick, S. W. An in Vivo Map of the Yeast Protein Interactome. Science 2008, 320, 1465-1470.

(9) Deeds, E. J.; Ashenberg, O.; Gerardin, J.; Shakhnovich, E. I. Robust protein-protein interactions in crowded cellular environments. Proceedings of the National Academy of Sciences 2007, 104, 14952-14957.

(10) Das, R. K.; Pappu, R. V. Conformations of intrinsically disordered proteins are influenced by linear sequence distributions of oppositely charged residues. Proceedings of the National Academy of Sciences of the United States of America 2013, 110, 13392-13397.

(11) Statt, A.; Casademunt, H.; Brangwynne, C. P.; Panagiotopoulos, A. Z. Model for disordered proteins with strongly sequence-dependent liquid phase behavior. 2019, 2327.

(12) Vacha, R.; Frenkel, D. Relation between Molecular Shape and the Morphology of SelfAssembling Aggregates: A Simulation Study. Biophys. J. 2011, 101, 1432 - 1439. 
(13) Šarić, A.; Chebaro, Y. C.; Knowles, T. P. J.; Frenkel, D. Crucial role of nonspecific interactions in amyloid nucleation. Proc. Natl. Acad. Sci. U.S.A. 2014, 111, 1786917874.

(14) Osmanović, D.; Rabin, Y. Effect of non-specific interactions on formation and stability of specific complexes. Journal of Chemical Physics 2016,

(15) Choi, J.-M.; Holehouse, A. S.; Pappu, R. V. Physical Principles Underlying the Complex Biology of Intracellular Phase Transitions. Annual Review of Biophysics 2020, 49, 107133, PMID: 32004090.

(16) Harmon, T. S.; Holehouse, A. S.; Rosen, M. K.; Pappu, R. V. Intrinsically disordered linkers determine the interplay between phase separation and gelation in multivalent proteins. eLife 2017, 6, e30294.

(17) Zhang, J.; Muthukumar, M. Simulations of nucleation and elongation of amyloid fibrils. The Journal of Chemical Physics 2009, 130, 035102.

(18) Hofweber, M.; Dormann, D. Friend or foe — post-translational modifications as regulators of phase separation and RNP granule dynamics. Journal of Biological Chemistry 2018

(19) Lukatsky, D. B.; Afek, A.; Shakhnovich, E. I. Sequence correlations shape protein promiscuity. Journal of Chemical Physics 2011, 135, 1-15.

(20) Sharma, R.; Raduly, Z.; Miskei, M.; Fuxreiter, M. Fuzzy complexes: Specific binding without complete folding. FEBS Letters 2015, 589, 2533-2542.

(21) Protter, D. S.; Parker, R. Principles and Properties of Stress Granules. Trends in Cell Biology 2016, 26, $668-679$. 
(22) Das, R. K.; Pappu, R. V. Conformations of intrinsically disordered proteins are influenced by linear sequence distributions of oppositely charged residues. Proc. Natl. Acad. Sci. U.S.A. 2013, 110, 13392-13397.

(23) Feric, M.; Vaidya, N.; Harmon, T. S.; Mitrea, D. M.; Zhu, L.; Richardson, T. M.; Kriwacki, R. W.; Pappu, R. V.; Brangwynne, C. P. Coexisting Liquid Phases Underlie Nucleolar Subcompartments. Cell 2016, 165, 1686-1697.

(24) Banani, S. F.; Lee, H. O.; Hyman, A. A.; Rosen, M. K. Biomolecular condensates: organizers of cellular biochemistry. Nature Reviews Molecular Cell Biology 2017, 18, 285.

(25) Tiwary, A. K.; Zheng, Y. Protein phase separation in mitosis. Current Opinion in Cell Biology 2019, 60, 92-98.

(26) Alberti, S.; Gladfelter, A.; Mittag, T. Considerations and Challenges in Studying Liquid-Liquid Phase Separation and Biomolecular Condensates. Cell 2019, 176, 419434.

(27) Ray, S. et al. $\alpha$-Synuclein aggregation nucleates through liquid-liquid phase separation. Nature Chemistry 2020,

(28) Aumiller, W. M.; Keating, C. D. Phosphorylation-mediated RNA/peptide complex coacervation as a model for intracellular liquid organelles. Nature Chemistry 2016,

(29) Ferreon, J. C.; Jain, A.; Choi, K.-J.; Tsoi, P. S.; MacKenzie, K. R.; Jung, S. Y.; Ferreon, A. C. Acetylation Disfavors Tau Phase Separation. International journal of molecular sciences 2018, 19, 1360.

(30) Alexander, C.; Toptygin, D.; Austin Elam, W.; TP, S.; Hilser, V. Phosphorylation Increases Persistence Length and End-to-End Distance of a Segment of Tau Protein. Biophys. J. 2016, 110, $362-371$. 
(31) Majumdar, A.; Dogra, P.; Maity, S.; Mukhopadhyay, S. Liquid-Liquid Phase Separation Is Driven by Large-Scale Conformational Unwinding and Fluctuations of Intrinsically Disordered Protein Molecules. Journal of Physical Chemistry Letters 2019, 10, 39293936.

(32) Shin, Y.; Brangwynne, C. P. Liquid phase condensation in cell physiology and disease. Science 2017, 357.

(33) Murthy, A. C.; Dignon, G. L.; Kan, Y.; Zerze, G. H.; Parekh, S. H.; Mittal, J.; Fawzi, N. L. Molecular interactions underlying liquid-liquid phase separation of the FUS low-complexity domain. Nature Structural and Molecular Biology 2019,

(34) Patel, A. et al. A Liquid-to-Solid Phase Transition of the ALS Protein FUS Accelerated by Disease Mutation. Cell 2015,

(35) Ranganathan, S.; Shakhnovich, E. I. Dynamic metastable long-living droplets formed by sticker-spacer proteins. eLife $\mathbf{2 0 2 0 ,}$

(36) Ma, B.; Nussinov, R. Molecular dynamics simulations of alanine rich $\beta$-sheet oligomers: Insight into amyloid formation. Protein Sci. 2002, 11, 2335-2350.

(37) Gillam, J. E.; MacPhee, C. E. Modelling amyloid fibril formation kinetics: mechanisms of nucleation and growth. J. Phys.: Condens. Matter 2013, 25, 373101.

(38) Hnisz, D.; Shrinivas, K.; Young, R. A.; Chakraborty, A. K.; Sharp, P. A. A Phase Separation Model for Transcriptional Control. Cell 2017, 169, 13 - 23.

(39) Ranganathan, S.; Maji, S. K.; Padinhateeri, R. Defining a Physical Basis for Diversity in Protein Self-Assemblies Using a Minimal Model. Journal of the American Chemical Society 2016, 138, 13911-13922. 
(40) Ghosh, S.; Ghosh, D.; Ranganathan, S.; Anoop, A.; P, S. K.; Jha, N. N.; Padinhateeri, R.; Maji, S. K. Investigating the Intrinsic Aggregation Potential of Evolutionarily Conserved Segments in p53. Biochemistry 2014, 53, 5995-6010, PMID: 25181279.

(41) Jha, N. N.; Anoop, A.; Ranganathan, S.; Mohite, G. M.; Padinhateeri, R.; Maji, S. K. Characterization of amyloid formation by glucagon-like peptides: role of basic residues in heparin-mediated aggregation. Biochemistry 2013, 52, 8800-10.

(42) Plimpton, S. Fast Parallel Algorithms for Short-Range Molecular Dynamics. J. Comput. Phys. 1995, 117, $1-19$.

(43) Bellesia, G.; Shea, J.-E. Effect of $\beta$-sheet propensity on peptide aggregation. J. Chem. Phys. 2009, 130.

(44) Bieler, N. S.; Knowles, T. P. J.; Frenkel, D.; Vácha, R. Connecting Macroscopic Observables and Microscopic Assembly Events in Amyloid Formation Using Coarse Grained Simulations. PLoS Comput. Biol. 2012, 8, e1002692.

(45) Sugita, Y.; Okamoto, Y. Replica-exchange molecular dynamics method for protein folding. Chemical Physics Letters 1999,

(46) Qi, R.; Wei, G.; Ma, B.; Nussinov, R. Methods in Molecular Biology; 2018. 